\title{
The Impact of Delayed Topology Information in Proactive Routing Protocols for MANETs ${ }^{*}$
}

\author{
Andres Medina and Stephan Bohacek \\ University of Delaware \\ Department of Electrical and Computer Engineering \\ Newark, DE 19716 \\ USA \\ \{medina,bohacek\}@ece.udel.edu
}

\begin{abstract}
In the design of many proactive routing protocols for MANETs, it is often assumed that topology information is disseminated instantly and error free. Exceptions include hazysighted routing, which deliberately delay the dissemination of topology information such that the average time from when a node learns about a change in topology depends on the distance from the node to where the topology information changed. However, the analysis of hazy-sighted routing neglects the impact of this delay. This paper explores the impact of delayed topology information to the probability of routing loops formation and and packet loss.
\end{abstract}

\section{Categories and Subject Descriptors}

C.2.2 [Computer-Communication Networks]: Network Protocols-Routing protocols

\section{General Terms}

algorithms, design, performance

\section{Keywords}

MANETs, proactive routing algorithms, routing loops

\section{INTRODUCTION}

Various researchers have studied the performance of routing protocols in MANETs. The authors in [1] for example, present a study in which the overhead of proactive protocols such as OLSR and DSDV is compared to the overhead

\footnotetext{
*This work was prepared through collaborative participation in the Collaborative Technology Alliance for Communications and Networks sponsored by the U.S. Army Research Laboratory under Cooperative Agreement DAAD1901-2-0011. The U.S. Government is authorized to reproduce and distribute reprints for Government purposes notwithstanding any copyright notation thereon.
}

Permission to make digital or hard copies of all or part of this work for personal or classroom use is granted without fee provided that copies are not made or distributed for profit or commercial advantage and that copies bear this notice and the full citation on the first page. To copy otherwise, to republish, to post on servers or to redistribute to lists, requires prior specific permission and/or a fee.

PE-WASUN'08, October 27-28, 2008, Vancouver, BC, Canada.

Copyright 2008 ACM 978-1-60558-236-8/08/10 ...\$5.00. in reactive routing protocols such as DSR and AODV. In this study and in many others analysis is made neglecting the delay associated with disseminating the topology information throughout the network. This delay can result in stale topology information, which can adversely impact the performance of the routing protocol.

Proactive routing protocols have the advantage that all the nodes maintain routes to every possible destination. Thus, a node that has stale topology information can successfully send the packet to the destination through the next hop listed in its outdated routing table. The next hop, as it is likely to be closer to the destination, may have more upto-date topology information of how to get to forward the packet to the destination. Therefore, when a node needs to send a packet using a proactive routing protocol, it needs only to check that the next hop is closer to the destination. Protocols such as hazy sighted link state routing [2] and fisheye routing [3] explicitly take advantage of this characteristic. In networks implementing these protocols, nodes maintain updated information about close by nodes. However they send topology updates at a low rate to nodes that are far away. In this manner, although the far away nodes may most likely have stale information, those nodes would have a vague idea of how to reach distant nodes. Thus, once the packet starts traveling through the network, it most likely will start using more correct routes to get to the destination. Note that while hazy-sighted routing and fish-eye routing explicitly assume that information about distance topology is not important for local routing decisions, other schemes might implicitly take advantage of this behavior. For example, probabilistic flooding schemes tend to reach distant nodes only after several tries.

However, some consequences of this feature have not been fully analyzed. This paper studies the formation of routing loops when not all the nodes have stored the same topology information. Specifically, the formation of loops of length two is studied, where the loop is between a node and its immediate next hop in a path. Such loops might occur when the next hop has current topology information and the node in question has stale information. The results of this study are used to model the more general problem of finding the probability of successful delivery of a packet, which also takes into account the probability of a transmission error at the MAC layer, the probability of a link failure, and the probability of disconnection between the two end nodes. The probability of having an error at the MAC layer for transmission of a unicast packet is out of the scope of this 
paper and is only mentioned. Also a simple approximation is used to calculate the probability of a link failure. The probability of disconnection between two given nodes has been studied in part in [4]. However in this paper results of a study of the disconnection problem are presented which disagree with the ones presented in [4].

Delayed information is caused by intirinsict characteristic of the flooding mechanism used to transmit routing information to all nodes (e.g, time interval between topology updates) or by errors in the transmission of the flood message caused by MAC layer issues. The first cause of delay cannot be avoided without changing the algorithm used for this task and is independent of the MAC protocol used. This type of delay will always be present, thus there will always be a nonzero probability of using erroneous information. When the effects of MAC are taken into account, the probability of using stale information to route packets is incremented. This paper shows how each cause of delay influences the performance of the routing algorithm in terms of the probability of successful delivery of a packet.

The results presented in this paper correspond to data obtained for a network with nodes randomly placed in space and a fixed transmission range. However it is also described which data is necessary to produce the same outputs for other network topologies.

The rest of this paper is organized as follows. Section 2 describes the possible causes of packet loss when proactive routing protocols are used and presents calculations for the probability of successful delivery of a packet. Sections 3 to 5 study each of the causes of packet failure. Section 6 presents the results. Finally, Section 7 concludes the paper.

\section{PROBABILITY OF SUCCESSFUL DELIV- ERY OF A PACKET}

The dynamic characteristic of MANETs implies the existence of a variety of ways in which a packet can be discarded while traveling from one source $S$ to a destination $D$. A packet may be dropped because there is no path stored in the routing tables of $S$ or an intermediate node $A$ to reach the destination node $D$. It also may be discarded as a consequence of a MAC error (i.e., repeated collisions). Another cause of packet drop arises when a link along the path is broken, and since the break has not yet been detected, a node attempts to transmit over it. Also as it is explained below, when a link breaks or is created, a loop may form temporarily, causing a short-term disconnection between $S$ and $D$. Thus, the probability of successful delivery over a link $i, P\left(S D_{i}\right)$ is given by

$$
\begin{gathered}
P\left(S D_{i}\right)=\left(1-P_{\text {NoPath }}\right) \times\left(1-P_{m e}\right) \times \\
\left(1-P_{\text {linkFail }}\right) \times\left(1-P_{\text {Loop }}\right),
\end{gathered}
$$

where $P_{\text {NoPath }}$ is the probability that there is no path to $D$ stored at the routing tables of the node transmitting the packet over link $i, P_{m e}$ is the probability of a MAC failure, $P_{\text {linkFail }}$ is the probability of a link failure, and $P_{\text {Loop }}$ the probability of having an 2-hop loop at the $i$-th link in the path, i.e., the $i$-th node along the path transmits the packet to a node that will forward the packet back to the $i$-th node. Although an n-hop loop can occur, in this paper only the 2-hop loop is considered.

The probability of successful delivery of a packet over a path of length $h$ is given by

$$
P(S D)=\prod_{i=1}^{h} P\left(S D_{i}\right)
$$

While past research efforts have modeled connectivity of networks, link breakage, and MAC error, modeling temporary loops has not been addressed. Clearly, a loop can only occur when a link breaks and there is an inconsistency in the routing information of nodes. The frequency of occurrence of link breaks and subsequent loops depends on the rate that links break and the rate that the routing algorithm disseminates topology information. Let $\mu$ be the rate at which links break and let $\lambda$ be the rate that each node generates and broadcasts new topology information. Define $K:=\lambda / \mu$ to be the average number of topology announcement generated between each link failure. Note that "speeding up time" so that links breaks at a rate of $2 \mu$ and topology announcements are generated at rate $2 \lambda$, does not change the value of $K$.

\section{PROBABILITY OF LOOP FORMING}

\subsection{Inconsistent Topology Information}

Denote $T(n ; K, \mu)$ the average time it takes for a node $n$ hops from the source of topology information to learn about topology changes. When all nodes transmit the topology update packet with infinite ttl (full flooding), $T(n ; K, \mu)$ is given by

$$
T(n ; K, \mu)=\left\{\begin{array}{ll}
\frac{1}{2 K \mu}, & \text { if } n>0 \\
0, & \text { if } n=0
\end{array} .\right.
$$

In hazy sighted routing [2], if broadcast delay is neglected, $T(n ; K, \mu)$ is given by

$$
T(n ; K, \mu)=\left\{\begin{array}{ll}
\frac{1}{K \mu} 2^{\left\lceil\log _{2} n\right\rceil-1}, & \text { if } n>0 \\
0, & \text { if } n=0
\end{array},\right.
$$

where $\lceil u\rceil$ is the largest integer larger than $u$. It is interesting to note that if the destination of a packet is $n$ hops away and $K>2^{\left\lceil\log _{2} n\right\rceil-1}$, then with a probability close to 1 there will be a link break along the path. Indeed, the premise of hazy-sighted routing is that information about the topology that is far away is not important, and hence nodes will often have incorrect information about the topology that is far away.

Let $D T(n, l ; K, \mu)$ be the average duration of time in which a node has information about a topology change that is $n$ hops away, but a node that is $l$ hops from the topology change has not learned about this information. Note that if topology information travels along the same paths as data packets (e.g., reverse path forwarding) and $n>l$, then $D T(n, l ; K, \mu)=0$. In the case of efficient flooding schemes that construct an overlay over which topology control messages are flooded might have $D T(n, l ; K, \mu)>0$ even when $n>l$. This paper focus on the case of hazysight-style flooding and full flooding where data packets and topology control messages follow the same paths, however, we include the possibility that $D T(n, l ; K, \mu)>0$ for $n>l$ for completeness.

If full flooding is used, then $D T(l-1, l ; K, \mu)$ is given by 


$$
D T(l-1, l ; K, \mu)=\left\{\begin{array}{ll}
\frac{1}{2 K \mu}, & \text { if } l=1 \\
0, & \text { otherwise }
\end{array} .\right.
$$

If hazy sighted is used and $l, n>0$, then $D T(n, l ; K, \mu)$ is given by

$$
D T(n, l ; K, \mu)=\frac{1}{K \mu} 2^{\left\lceil\log _{2} n\right\rceil-1}\left(2^{\left\lceil\log _{2} l\right\rceil-\left\lceil\log _{2} n\right\rceil}-1\right) .
$$

Of particular interest is the case where $n=l-1$ and $l \geq 1$, that is, where there is topology information between neighboring nodes is inconsistent. In this case, when a link breaks, topology information becomes inconsistent for an interval of time given by

$$
\begin{aligned}
D T(l-1, l ; K, \mu) & =\left\{\begin{array}{cc}
\frac{1}{K \mu} 2^{\left\lceil\log _{2}(l-1)\right\rceil-1} \times \\
\left(2^{\left\lceil\log _{2} l\right\rceil-\left\lceil\log _{2}(l-1)\right\rceil}-1\right), & \text { if } l>1 \\
\frac{1}{2 K \mu}, & \text { if } l=1
\end{array}\right. \\
& =\left\{\begin{array}{cc}
\frac{l-1}{2 K \mu}, & \text { if } l=2^{p}+1, \\
\frac{1}{2 K \mu}, & \text { if } l=1,2, \ldots\} \\
0 & \text { otherwise }
\end{array}\right.
\end{aligned}
$$

$D T(l-1, l ; K, \mu)$ is the duration of inconsistent topology information when a link that is $l$ hops away breaks. The duration between link breaks is $1 / \mu$. Thus, the fraction of time when topology information is inconsistent is

$$
\frac{D T(l-1, l ; K, \mu)}{\frac{1}{\mu}} .
$$

MAC and physical layer errors can keep a node from receiving information about a topology change. If a node cannot receive a topology update successfully, it must wait until the next topology update for another opportunity to obtain information about the change in topology. Let $P_{f e}(l)$ the probability of failure to received a flooding message by a node $l$ hops away from the source. Also let $P_{i f}(l)$ the probability of topology inconsistency in the forward direction, that is a node $l-1$ hops away from the source of the flooding message received the information and its neighbor at $l$ did not receive the information.

Consider first the case when the routing algorithm uses full flooding to disseminate topology updates. Denote $p_{i c}(i, l)$ the probability that $i$ topology updates are required to solve a particular information inconsistency between a node $l-1$ hops from the originator of the information and its neighbor at $l$ hops. $p_{i c}(i, l)$ is given by

$$
p_{i c}(i, l)=\left\{\begin{array}{ll}
P_{f e}(1)^{i-1}\left(1-P_{f e}(1)\right), & \text { if } l=1 \\
P_{i f}(l) P_{f e}(l)^{i-1}\left(1-P_{f e}(l)\right), & \text { if } l>1
\end{array} .\right.
$$

$D T(l-1, l ; K, \mu)$ is then found using

$$
D T(l-1, l ; K, \mu)=\sum_{i=1}^{\infty} D T(l-1, l, i ; K, \mu) p_{i c}(i, l),
$$

where $D T(l-1, l, i ; K, \mu)$ is given by

$$
D T(l-1, l, i ; K, \mu)=\left\{\begin{array}{ll}
\frac{1}{K \mu}\left(\frac{1}{2}+i-1\right), & \text { if } l=1 \\
\frac{1}{K \mu}(i-1), & \text { if } l>1
\end{array} .\right.
$$

Thus $D T(l-1, l ; K, \mu)$ is given by:

$$
D T(l-1, l ; K, \mu)=\left\{\begin{array}{ll}
\frac{1}{K \mu}\left(\frac{1}{1-P_{f e}(1)}-\frac{1}{2}\right), & \text { if } l=1 \\
\frac{P_{i f}(l) P_{f e}(1)}{K \mu\left(1-P_{f e}(1)\right)}, & \text { if } l>1
\end{array} .\right.
$$

For the case of hazy sighted, when $l=2^{p}+1$, the node $V$ at $l-1$ hops, receives topology updates twice as fast as the node $W$ at $l$ hops. Let $E$ be the event of $V$ receiving the topology message in a topology update with $t t l=l-1$, i.e., cannot reach $W$. Note that if it is assumed that $W$ never receives a topology update before $V$, then

$$
P(E)=\left\{\begin{array}{cc}
1, & \text { if } l=1 \\
\frac{1}{2}, & \text { if } l=2^{p}+1, \\
0, & \text { otherwise }
\end{array} .\right.
$$

$D T(l-1, l, i ; K, \mu \mid E)^{1}$ is given by

$$
\begin{aligned}
& D T(l-1, l, i ; K, \mu \mid E)= \\
& \left\{\begin{array}{cc}
\frac{1}{K \mu}\left(\frac{1}{2}+i-1\right), & \text { if } l=1 \\
2 \frac{l-1}{K \mu}\left(\frac{1}{2}+i-1\right), & \text { if } l=2^{p}+1,
\end{array}\right.
\end{aligned}
$$

and $D T(l-1, l, i ; K, \mu \mid \bar{E})$ is

$$
D T(l-1, l, i ; K, \mu \mid \bar{E})=\frac{2^{\left\lceil\log _{2} l\right\rceil}}{K \mu}(i-1),
$$

$p_{i c}(i, l \mid E)$ is given by

$$
p_{i c}(i, l \mid E)=P_{f e}(l)^{i-1}\left(1-P_{f e}(l)\right)
$$

and $p_{i c}(i, l \mid \bar{E})$ is

$$
p_{i c}(i, l \mid \bar{E})=P_{i f}(l) P_{f e}(l)^{i-1}\left(1-P_{f e}(l)\right)
$$

Then $D T(l-1, l ; K, \mu)$ is given by

$$
\begin{aligned}
& D T(l-1, l ; K, \mu)= \\
& P(E) \sum_{i=1}^{\infty} D T(l-1, l, i ; K, \mu \mid E) p_{i c}(i, l \mid E)+ \\
& (1-P(E)) \sum_{i=1}^{\infty} D T(l-1, l, i ; K, \mu \mid \bar{E}) p_{i c}(i, l \mid \bar{E}) . \\
& \qquad \begin{array}{cl}
\frac{(l-1)}{K \mu}\left(\frac{1}{\left(1-P_{f e}(l)\right)}-\frac{1}{2}\right. & \text { if } l=2^{p}+1, \\
\left.+P_{i f}(l) \frac{P_{f e}(l)}{1-P_{f e}(l)}\right), & p \in\{1,2, \ldots\} \\
2^{\left\lceil\log _{2} l\right\rceil \frac{P_{i f}(l) P_{f e}(l)}{K \mu\left(1-P_{f e}(l)\right)},} & \text { otherwise }
\end{array}
\end{aligned}
$$

The probabilities $P_{f e}$ and $P_{i f}$ are closely related with the probability of a node $W$ receiving a packet from its neighbor $V, p_{E r r}$. Intuitively, the event of successful delivery of a broadcast packet from $V$ to $W$ is closely correlated with the same event for $V$ and neighbors of $V$ close to $W$. This correlation is a consequence of the MAC and physical layer

\footnotetext{
${ }^{1}$ For simplicity, cases with probability zero are not consid-
} ered. 


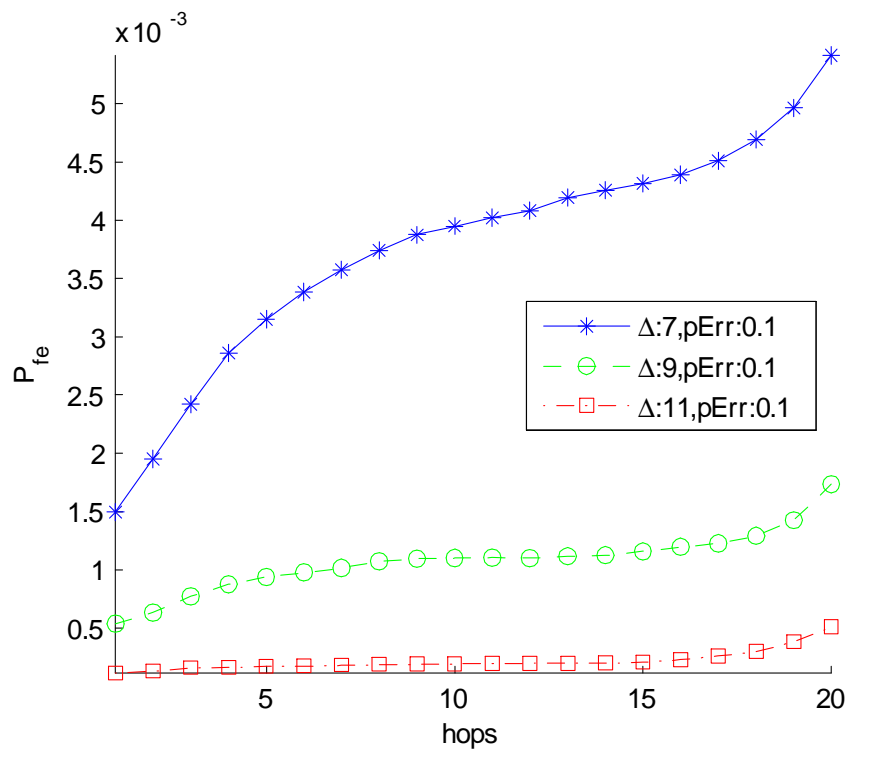

Figure 1: Probability that a node fails to receive a topology update message in networks of $20 \times 20$ transmission ranges and different node degree. $\mathrm{X}$ axis is the number of hops that separate the node from the originator of the topology packet.

and thus it is out of the scope of this work. Therefore, we will assume in this paper that the probabilities for these events are independent. Future work should focus on the modelling of $p_{E r r}$. The Figures 1 and 2 shows the approximated values of $P_{f e}(l)$ obtained from $4 \times 10^{5}$ simulations in networks of size $20 \times 20$ transmission ranges for different $p_{E r r}$ and different degrees, respectively. Figures 3 and 4 shows the approximated values of $P_{i f}(l)$ calculated from data of $4 \times 10^{5}$ simulations in the same scenarios.

\subsection{Probability of a loop forming when a link is broken}

Consider the situation depicted in Fig. 5. A node $A$ sends data to another node $D$ through its neighbor $B$. Node $D$ is $h$ hops away from $A$, with $h \geq 2$. Clearly node $B$ is $h-1$ hops away from $D$. Consider now a link breakage at the $k$-th hop from $A$ in the path from $A$ to $D$. It is reasonable to expect that node $B$ receives this information of change in the topology of the network before $A$. During $D T(k-2, k-1 ; K, \mu)$, the nodes $A$ and $B$ would have different topology information. A routing loop between $A$ and $B$ would then occur, if $B$ determines from its new topology information that the best path to go from $B$ to $D$ is through $A$.

Denote $h_{A \rightarrow D, \sim k}$ the length of the path from $A$ to $D$ after the $k$-th link breaks. Also denote $h_{B \rightarrow D, \sim A, \sim k}$ the length of the path from $B$ to $D$ that avoids link $k$ and node $A$. During $D T(k, k-1 ; K, \mu)$, three cases are of interest.

1. If $h_{B \rightarrow D, \sim A, \sim k}-1>h_{A \rightarrow D, \sim k}$ then $B$ 's shortest path to $D$ is through $A$ and hence $B$ will forward its packet through $A$, creating a loop.

2. If $h_{B \rightarrow D, \sim A, \sim k}-1=h_{A \rightarrow D, \sim k}$ then $B$ has more than one shortest path to reach $D$, and one of these shortest

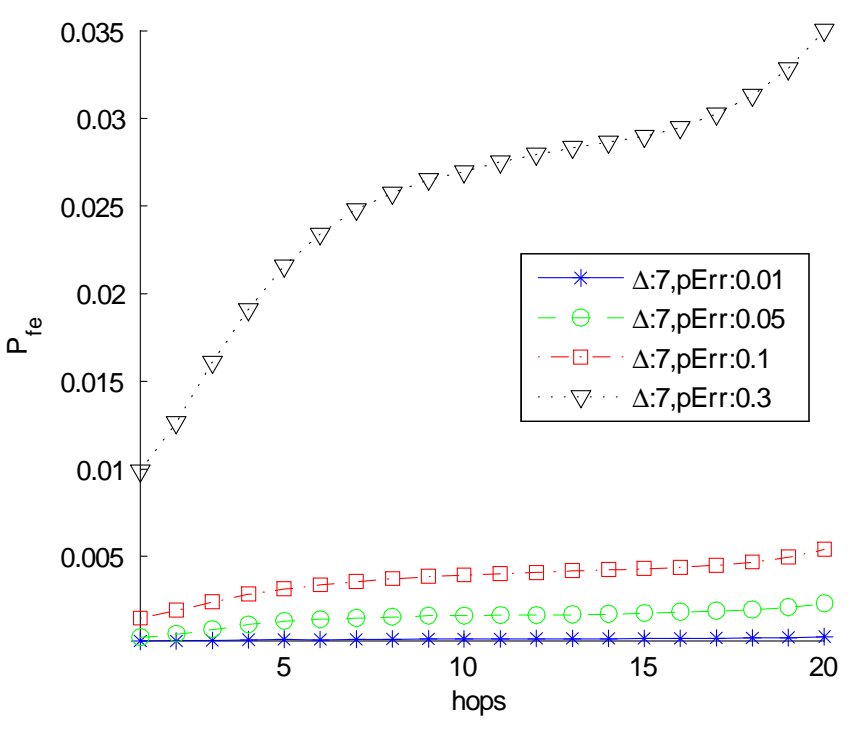

Figure 2: Probability that a node fails to receive a topology update message in networks of $20 \times 20$ transmission ranges and different probability of error. $\mathrm{X}$ axis is the number of hops that separate the node from the originator of the topology packet.

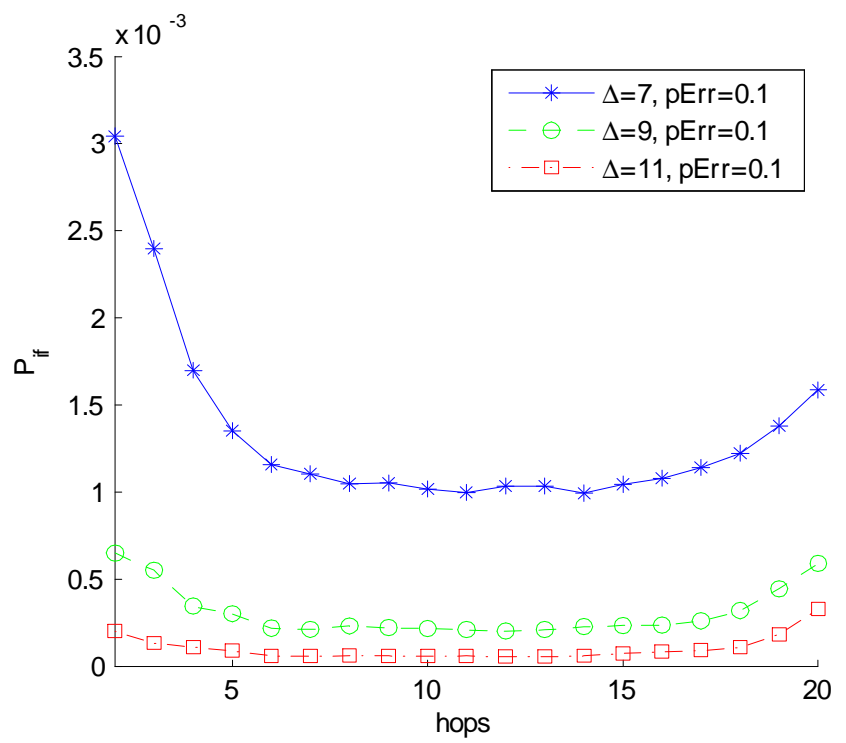

Figure 3: $P_{i f}(n)$ vs $n$. Probability that a node $n-1$ hops from the Originator of the topology update receives flood message, but its neighbor at $n$ hops fails to received it. Networks size is $20 \times 20$ transmission ranges. $P_{i f}(n)$ is shown for different node degrees. 


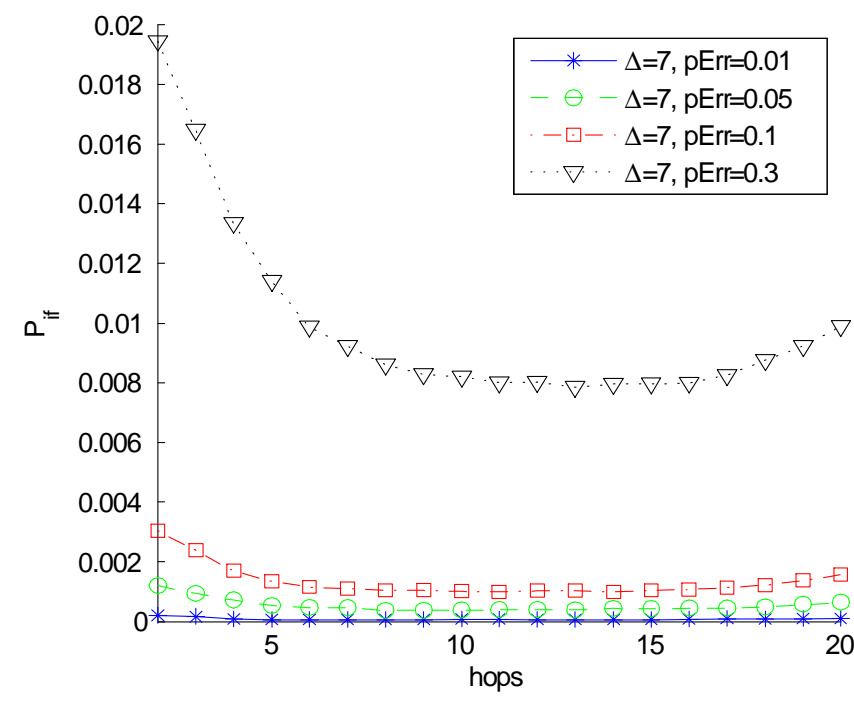

Figure 4: $P_{i f}(n)$ vs $n$. Probability that a node $n-1$ hops from the Originator of the topology update receives flood message, but its neighbor at $n$ hops fails to received it. Networks size is $20 \times 20$ transmission ranges. $P_{i f}(n)$ is shown for different error probabilities.

\section{Loop forms during} topology information imbalance

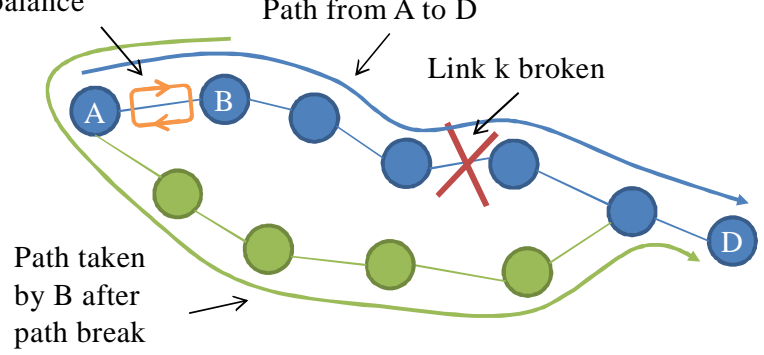

to reach $\mathrm{D}$

Figure 5: Loop forming process when a link breaks. Node $A$ has stale topology information. Node $B$ has updated information. A loop forms because of information disparity paths is through $A$. In this case there would be a loop if $B$ chooses the path that goes through $A$.

3. Finally, a loop is not created if $h_{B \rightarrow D, \sim A, \sim k}-1<$ $h_{A \rightarrow D, \sim}$, i.e., $B$ has a path to $D$ that is shorter than any path through $A$.

It is important to note that loops are unlikely to occur in dense networks. To see this, note that the length of path from $B$ to $D$ via $A$ cannot be any shorter than $h_{B \rightarrow D}+2$ (otherwise $A$ would not forward packets to $B$ ). Thus, in order for a link break to result in a loop, $B$ must not have any alternative paths of length $h_{B \rightarrow D}$, and $h_{B \rightarrow D}+1$. Since dense networks provide many alternative paths with similar length, loops are unlikely to occur in dense networks.

In case 2, a loop may form, but not necessarily. Specifically, in this case, there is an alternative path from $B$ through a node $C$ to $D$, besides the path through $A$. A protocol might implement a logical tweak to presumably avoid some loop by forcing node $B$ to transmit the packets through $C$ if it received a packet from $A$. However, the cause of inconsistent topology information between $A$ and $B$ is also likely to result in a topology information inconsistency between $C$ and $B$. Therefore, we assume that a loop will occur when either case 1 or case 2 arises.

Denote $P_{L}(k ; h)$ the probability of a loop forming, given that the information between the node at the $(k-1)$-th hop and the node at the $k$-th hop is inconsistent and the path length is $h$ hops. The following experiment was repeated $13 \times 10^{6}$ to recollect data for the estimation of $P_{L}(k ; h)$. The network size was set to $20 \times 20$ transmission ranges. Figure 6 shows the calculated values of $P_{L}(k ; h)$, as a function of $k$ for different path lengths and average node degrees, $\Delta$. As expected, Figure 6 shows that denser networks (i.e., large values of $\Delta$ ) have a lower probability of loops. Also, the probability of a loop occurring is smaller when the link break is further away. This result agrees with the intuition that topology changes that are far away are not important.

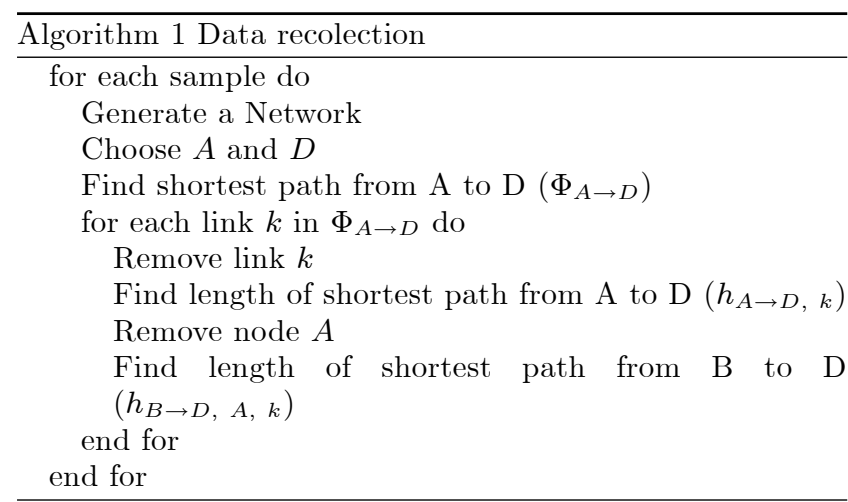

The probability of having a loop occurring at any particular moment is the probability of having a loop when there is inconsistent topology information between the current node that is transmitting the packet and the next node in the path multiplied by the probability of actually having this inconsistency, which is given by (2). Thus, letting $P_{\text {loop }}(k$ down; $h)$ be the probability of having a loop in the first link (i.e., a node will forward that packet to a neighbor, which will forward it back) and a link breakage at the $k$-th 


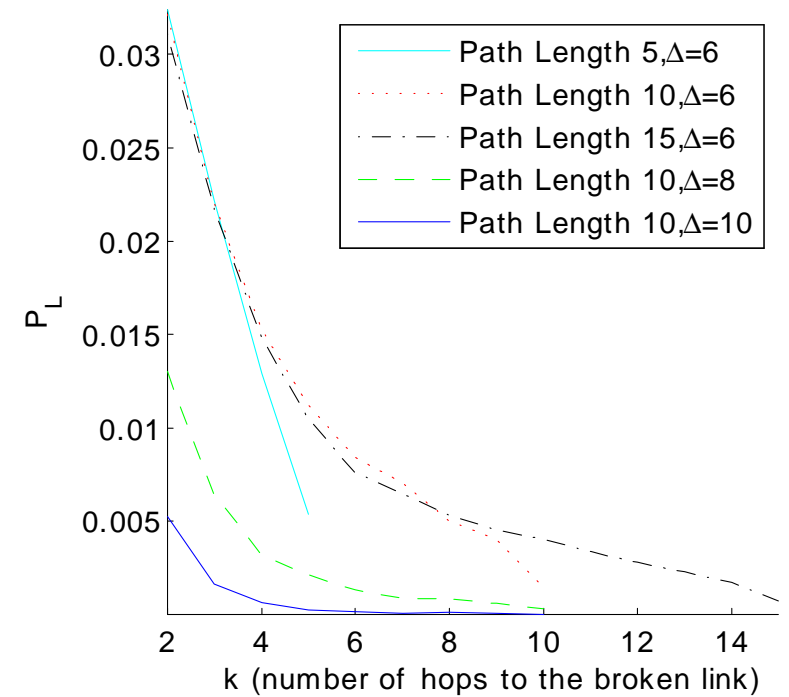

Figure 6: Probability of having a loop of length two, given that a link $k$ hops away has broken, the source is unaware of the failure and the neighbor that the source forwards the packet to is aware of the link break. This probability is show for a range of node density, where $\Delta$ is the average number of neighbors.

link of a path of length $h$ is given by

$$
P_{\text {loop }}(k \text { down } h)=P_{L}(k ; h) \frac{D T(k-2, k-1 ; K, \mu)}{\frac{1}{\mu}},
$$

with $k \in[2, h]$.

\subsection{Probability of loop forming when a link is created}

It is also possible that a loop can form when a link comes up. Referring to Figure 5, when the link marked with the $X$ comes up, if $A$ learns that the link is up before $B$, then $A$ will forward packet to $B$, and $B$ will forward the packet back to $A$. Note that this type of loop requires that $A$ learn topology information before $B$ while $B$ is closer to the source of topology information. Clearly if topology information messages follow shortest paths, then this type of information inconsistency cannot occur. However, as mentioned above, if topology control messages are spread over overlays (e.g., [5]), then this type of information inconsistency can occur.

Denote by $P_{\text {Loop }}(k$ up; $h)$ the probability of having a loop at the first link in a path of length $h$ and the $k$-th link in the route just created. $P_{\text {Loop }}(k$ up; $h)$ is given by

$$
P_{\text {Loop }}(k \text { up } ; h)=P_{L}(k ; h) \frac{D T(k-1, k-2 ; K, \mu)}{\frac{1}{\mu}},
$$

where $D T(k-1, k-2 ; K, \mu)=0$ if topology messages follow shortest paths. Note that $P_{L}(k ; h)$ used above is the same one used in (3). That is, given the correct type of information inconsistency, the probability of a loop forming when removing a link is the same as a loop forming when adding a link. This is justified by the reversibility of the mobility. Thus, in the forward case, loops are caused when a link breaks causes the cases 1 or 2 to occur. In the reverse case, a loop will occur when a link forms and the topology was in case 1 or 2 . Thus, in either case, the probability of a loop occurring is the same.

Combining (3) and (4), the probability that the transmission to the next hop fails because there is a loop, given that the remaining path is of length $h$ is given by

$$
P_{\text {Loop }}(h)=\sum_{k=2}^{h}\left(P_{\text {Loop }}(h \text { and } k \text { down })+P_{\text {Loop }}(h \text { and } k \text { up })\right) .
$$

\section{PROBABILITY OF LINK FAILURE}

As explained above, a node discards a packet if the link to the next hop is no longer present. Nodes relay on a neighbor detection mechanism to detect broken links. The average time a node needs to detect a broken link, depends on the specification of the chosen neighborhood discovery procedure. If hello messages are used for this detection mechanism, usually some number $n$ of missed hellos from a neighbor are necessary to declare a link as broken. In general, a node maintains a link in the link set without receiving any updates for a time $t_{R S}$ specified by the algorithm. The probability of link failure is then given by

$$
P_{\text {linkFail }}=\frac{\left(\frac{1}{2 \lambda_{H}}+t_{R S}\right)}{\frac{1}{\mu}}
$$

where $\lambda_{H}$ is the hello packet rate.

\section{PROBABILITY OF NO PATH IN THE TA- BLE}

To calculate the probability that two nodes are disconnected, simulations were run for various densities. Figure 7 shows this probability as a function of the node degree $\Delta$. Each point in Figure 7 is calculated using approximately $1.6 \times 10^{6}$ samples. Note that there is a distinction between a node not having a path in its table and the network actually being partitioned. However, here we approximate the probability that there is no path in the table with the probability that two nodes are disconnected, thus for the $k$-th link in a path, with $k \geq 2, P_{\text {NoPath }}=0$.

\section{RESULTS}

For simplicity, in this section it is assumed an ideal MAC layer protocol that transmits all unicast packets, i.e., $P_{m e}=$ 0 . Also it is assumed that the link detection mechanism is perfect and detects the link breaks immediately, thus $P_{\text {linkFail }}=0$. However all calculations can be easily modified to account for these errors.

Figure 8 show the probability of successful delivery of a packet $P(S D)$ as a function of the average number of topology updates sent per link break in a network with nodes having an average degree $\Delta=7$ using hazy sighted. Figure 8 shows $P(S D)$ for different path lengths. Figure 9 shows the corresponding values for the full flooding case. As expected, the shorter path has lower probability of errors. Also as expected full flooding is more robust than hazy sighted. It can be noted, that when only the probability of disconnection is considered, $P(S D)$ is constant, as the packet is only dropped at the first link. When loops are considered, 


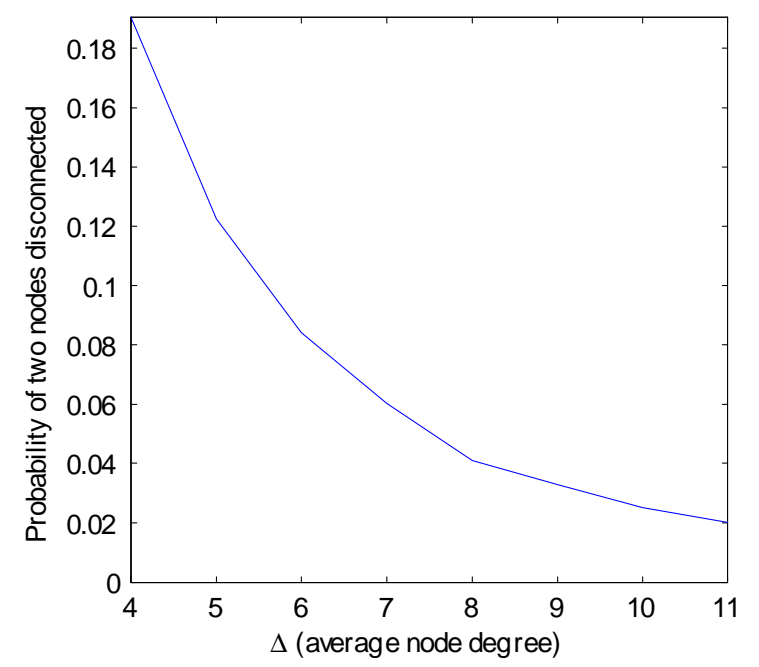

Figure 7: Probability that there is no path that connects two randomly selected nodes in a network with degree $\Delta$.

$P(S D)$ is significantly lower for small values of $K$. As expected, the probability of loops decreases as $K$ increases. Thus, for large $K$, the probability of loops can be neglected. Figure 10 shows $P(S D)$ for various values of node degree. It can be seen that loops are more frequent in low density topologies. Figure 11 shows $P(S D)$ for different $p_{E r r}$. It can be noted that even a very huge error of 0.5 does not change the result substantially.

\section{CONCLUSIONS}

In this paper it was shown how stale topology information impacts the probability of successful delivery of a packet. A trade-off is highlighted between increasing this probability and reducing the overhead of the routing protocol. It was shown that the probability of loops cannot be neglected if the rate of topology updates is low compared to node mobility.

Future work should also consider how stale topology information impact routing overhead, caused by the use of suboptimal routes.

\section{Disclaimer}

The views and conclusions contained in this document are those of the authors and should not be interpreted as representing the official policies, either expressed or implied, of the Army Research Laboratory or the U. S. Government.

\section{REFERENCES}

[1] L. Viennot, P. Jacquet, and T. H. Clausen, "Analyzing control traffic overhead versus mobility and data traffic activity in mobile ad-hoc network protocols," Wirel. Netw., vol. 10, no. 4, pp. 447-455, 2004.

[2] C. Santivanez and R. Ramanathan, "Hazy sighted link state (HSLS) routing: A scalable link state algorithm," BBN Tech-

nologies, Tech. Rep. 1301, Aug 2001. [Online]. Available: http://www.ir.bbn.com/documents/techmemos/TM1301.pdf

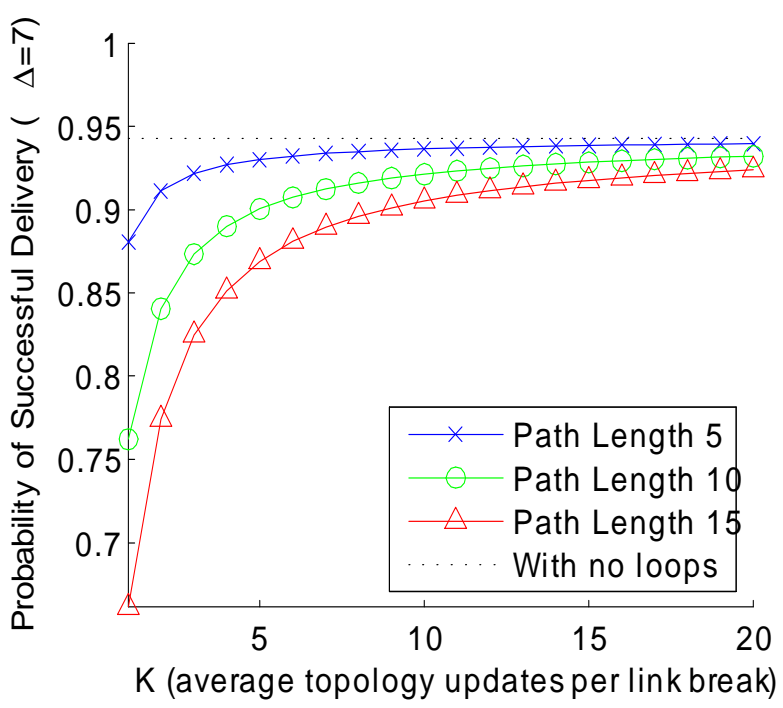

Figure 8: Probability of successfully delivering a packet vs. the average number of topology updates sent per link break when the average node degree is 7 and Hazy Sighted is used. Dotted line is the probability of successfully delivering a packet if no loops are considered. Also $P_{m e}=P_{\text {linkFail }}=$ $p_{\text {err }}=0$.

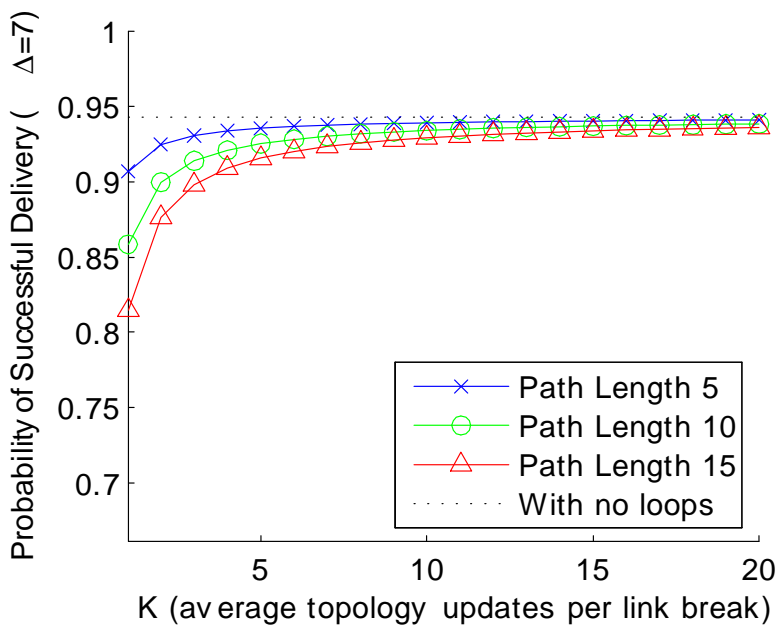

Figure 9: Probability of successfully delivering a packet vs. the average number of topology updates sent per link break when the average node degree is 7 and full flooding is used. Dotted line is the probability of successfully delivering a packet if no loops are considered. Also $P_{m e}=P_{\text {linkFail }}=$ $p_{\text {err }}=0$. 


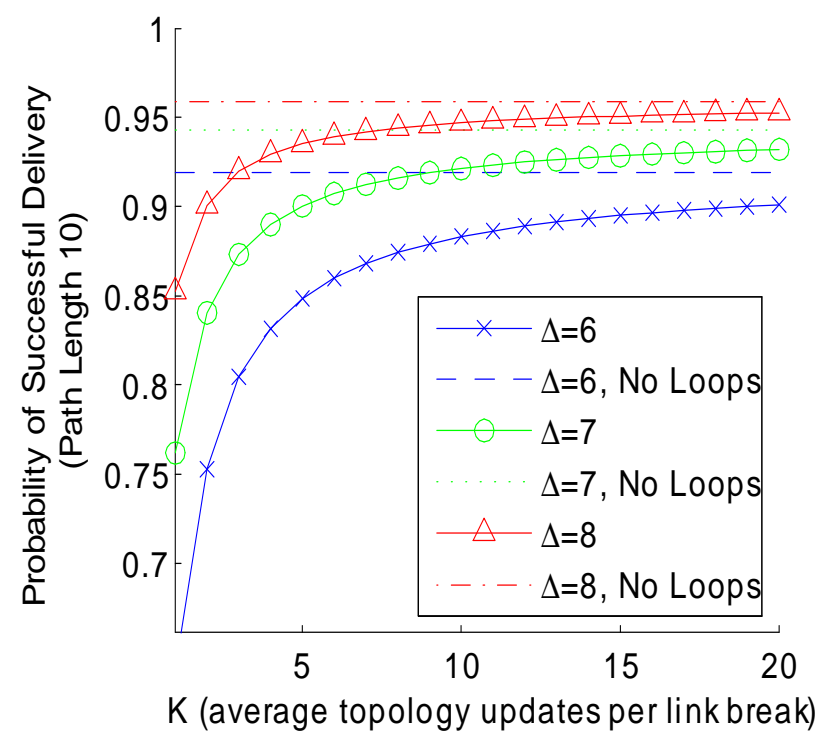

Figure 10: Probability of successfully delivering a packet vs. the average number of topology updates sent per link break for a path of length 15 and Hazy Sighted is used. Constant probability lines indicate the probability of successfully delivering a packet if no loops are considered. Also $P_{m e}=P_{\text {linkFail }}=p_{\text {err }}=0$.

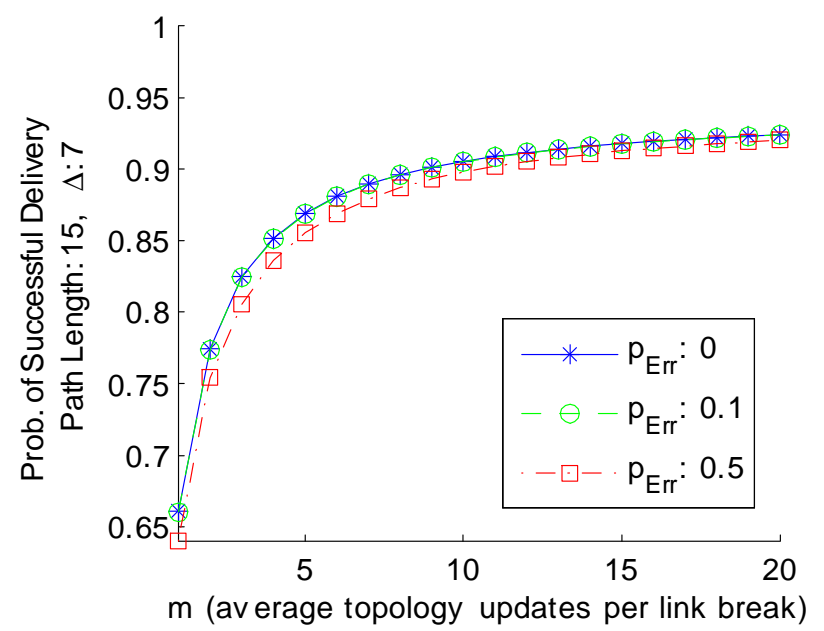

Figure 11: Probability of successfully delivering a packet vs. the average number of topology updates sent per link break when the average node degree is 7 , path length is 15 and Hazy Sighted is used. Also $P_{m e}=P_{\text {linkFail }}=0$.
[3] G. Pei, M. Gerla, and T.-W. Chen, "Fisheye state routing in mobile ad hoc networks," in ICDCS Workshop on Wireless Networks and Mobile Computing, 2000, pp. D71-D78.

[4] C. Bettstetter, "On the connectivity of ad hoc networks," The Computer Journal, vol. 47, no. 4, pp. 432-447, April 2004.

[5] W. Peng and X. Lu, "Efficient broadcast in mobile ad hoc networks using connected dominating sets," Journal of Software, no. 10, pp. 529-536, 1999. 